\title{
La juventud en España
}

\author{
M. ㄹ JOSÉ AGUILERÅ
}

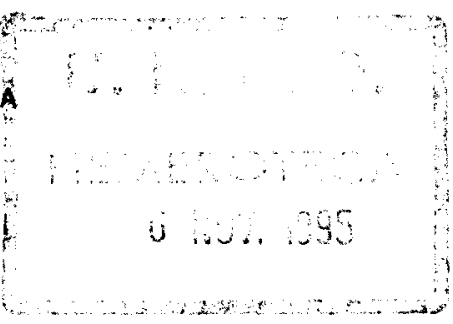

\section{INTRODUCCIÓN}

Existe actualmente, en España, una gran preocupación, entre los profesionales de la Geografía, por temas demográficos tan interesantes como los nuevos movimientos migratorios, el envejecimiento de la población o el descenso de la tasa de natalidad; sin embargo, es difícil encontrar trabajos dedicados al estudio de otro grupo o conjunto de personas cuya característica fundamental es "ser joven", o, lo que es lo mismo, estar en una etapa de la vida comprendida entre unos años determinados. Este colectivo presenta un volumen mayor y una problemática distinta, en algunas parcelas, de la que hasta ahora se conocía. Además, los problemas y las necesidades que presenta la juventud difieren, por su volumen y peculiaridades, de los que tenian las generaciones pasadas. La preocupación social por los fenómenos asociados a la juventud: educación, paro, salud, actitudes ante relaciones sexuales, políticas, sociales, divorcio, aborto, drogadicción, accidentes de tráfico, etc., es, hoy en día, tan importante como la inquietud por la problemática planteada por otros conjuntos de la población, y hace necesarias unas medidas políticas de planificación que prevean y prevengan las necesidades y problemas de los mismos.

Todo esto me llevó a pensar en la oportunidad de llamar la atención sobre este colectivo, para incluirlo, como objeto de estudio, en la investigaciones de los geógrafos preocupados por los temas de la población.

Es necesario conocer su volumen, su peso proporcional en el conjunto de la población, su proporción respecto a otros grupos de edad, su evolución, su distribución espacial, e incluso compararla con la de la Unión Europea, para establecer medidas que tiendan a solucionar o, al menos, a dirigir y tratar de paliar los problemas que se planteen. Aquellos espacios 
regionales donde las proporciones de jóvenes sean mayores serán los que tendrán que destinar más esfuerzo a la resolución de los problemas que plantea la juventud, simplemente por el hecho de ser mayor su cuantía. Además, se ha de ver cuáles son los prioritarios y sobre todo se habrán de analizar sus causas para corregirlas, en la medida de lo posible. El propósito de este artículo no es tan ambicioso como podría desprenderse de lo dicho hasta aquí; si no que se trata, simplemente, de cuantificar y estudiar la evolución y distribución espacial de los jóvenes españoles en estos años finales del siglo xx.

\section{VOLUMEN, EVOLUCIÓN Y DISTRIBUCIÓN ESPACIAL DE LA POBLACIÓN JUVENIL ESPAÑOLA A FINALES DEL SIGLO XX}

No todos los investigadores sociales se refieren a los mismos grupos de edad cuando hablan de población joven; esa diferencia está, sobre todo, en función del aspecto demográfico que vayan a estudiar. Los demógrafos y geógrafos humanos, cuando estudian la población del mundo, país, región etc., suelen clasificar los grupos de edad de la población que pretenden analizar, como sigue:

Jóvenes, personas comprendidas entre 0 y 14 años, o bien entre 0 y 19 años; adultos, quienes tienen entre 15 y 59 años, 15 y 64, 20 y 59 o 20 y 64 años; y por último, ancianos, cuando han cumplido los $60 \circ 65$ años. Los resultados proporcionales entre estos grupos de edad vienen a dar una idea de la juventud que tiene cada espacio geográfico que se esté analizando. Ello se debe a los criterios que se utilizan como base diferencial: interesa conocer las posibilidades de reproducción, actividad, estudios, etc; y además, se han de unificar esos criterios para poder englobar a todos los paises del mundo. Sin embargo, si se tratase de estudiar esos grupos de edad de forma independiente, las barreras de edad que limitarían estas etapas vitales serían diferentes. $\mathrm{Si}$, a lo largo de la vida media de una persona, quisiéramos señalar la edad correspondiente a su juventud biológica, es más probable que se diferenciasen, dentro de la edad adulta, los años que tras la pubertad preceden a la madurez, es decir, entre los 15 y 20 años. Sin embargo, razones de tipo social, más que biológico, llevan a los científicos sociales a establecer una etapa más larga: de 15 a 24 , de 16 a 24 o 29 años y de 16 a 30 años, fundamentalmente en países del ámbito occidental y desarrollado de todo el mundo. Si bien el año inicial ofrece menos dudas, el año final de esa etapa parece resultar menos claro. Hasta hace poco tiempo, se consideraban los 24 años como tope de esa fase biológica, sin embargo, actualmente, está mucho más 
aceptada la edad de 29 años como límite para que a una persona se le pueda llamar joven. Las razones son muy variadas:

En primer lugar, la prolongación de los años de vida para un gran número de personas. Esto produce el aumento de los años comprendidos en cada una de las etapas del ciclo biológico. Niñez, de 0 a 14 años; juventud, de 15 a 29 años; madurez, de 30 a 65 ó 70 años, y, por último, senectud o vejez, de esos años en adelante.

En segundo lugar, las actuales circunstancias económicas, sobre todo en las grandes ciudades, conducen a un aumento en la edad de emancipación de los jóvenes, una edad más tardía a la hora de casarse, etc., que recomienda el clasificar a la juventud entre los 15 y los 29 años, o desde los 16 a los 30 , si el estudio se centra en el aspecto laboral de la misma, dado que la legislación vigente establece los 16 años como la edad inicial para poder trabajar.

Por último, el alargamiento de la enseñanza básica, obligatoria hasta los 16 años, podría ser también un nuevo factor a tener en cuenta, aunque no esté aún en vigor al haberse retrasado la implantación de la LOGSE. Cuando la etapa de enseñanza obligatoria llegue a los 16 años, nos encontraremos con una doble razón para establecer en esa edad el final de la niñez, o, si se prefiere, el final de la etapa de mayor dependencia de los hijos.

El Instituto de la Juventud publicó en 1993, además del ya tradicional "Informe Juventud en España", otro volumen, "Juventud en cifras 1992", en cuyos datos nos vamos a basar para la elaboración de los mapas y gráficos objeto del presente trabajo. A partir de ellos trataremos de obtener las causas y consecuencias que esa distribución y proporción nos sugieran.

El volumen total de españoles que tenían, en 1991, entre 15 y 29 años, era de 9.621.228. Su distribución por Comunidades Autónomas no es homogénea, sino que existen bastantes diferencias, como sucede con otros muchos aspectos geográficos. En cifras absolutas es Andalucía la que tiene un mayor volumen (1.810.879), seguida de Cataluña (1.463.049) y Madrid (1.269.585). La Comunidad Valenciana ocupa el cuarto lugar con 960.519 jóvenes. Galicia, Castilla-León y País Vasco superan el medio millón de jóvenes, y próximos a esa cifra están Canarias y Castilla-La Mancha. Aragón, Asturias y Extremadura superan los 200.000, y no llegan a esa cifra, pero superan los 100.000, Baleares, Navarra y Cantabria. Sólo La Rioja está por debajo de esta cantidad (58.646). 
CUADRO I

\begin{tabular}{cccccc}
\hline & EDAD & $15-19$ & $20-24$ & $25-29$ & TOTAL NACIONAL \\
\hline Censo & 1960 & 2.434 .023 & 2.267 .697 & 2.446 .739 & 30.528 .539 \\
" & 1970 & 2.709 .336 & 2.548 .753 & 2.239 .480 & 34.040 .657 \\
" & 1981 & 3.263 .312 & 2.942 .178 & 2.537 .428 & 37.683 .363 \\
« & 1991 & 3.319 .480 & 3.212 .665 & 3.089 .083 & 38.872 .278 \\
\hline
\end{tabular}

Casi la cuarta parte de la población española en 1991 (24,75\%) tenía entre 15 y 29 años, lo que supone una gran proporción, tanto si se refiere a los propios grupos de edad de esa población (15 años suponen el $25 \%$ ), como si la comparamos con la proporción de dichos grupos en el conjunto de la Unión Europea (23,6\%). La citada publicación del Instituto de la Juventud daba para la C.E.E., en 1989, proporciones por debajo de la española en todos los países. A España, con un $25,02 \%$ en ese año, le seguían Portugal $(24,81 \%)$, Holanda $(24,62 \%)$, Italia $(24,15 \%)$ e Irlanda $(24,06 \%)$. El resto de los países estaba por debajo, siendo Grecia $(22,07 \%)$ y Luxemburgo $(22,22 \%)$ los que presentaban la menor proporción de jóvenes.

Vistos los cuatro últimos censos de población, es precisamente el último, 1991, el que presenta mayor proporción de jóvenes comprendidos entre los 15 y 29 años, siendo 1970 el que alcanzó menor porcentaje $(22,03 \%)$ (Gráfico no 1). Si consideramos su nacimiento, esta población

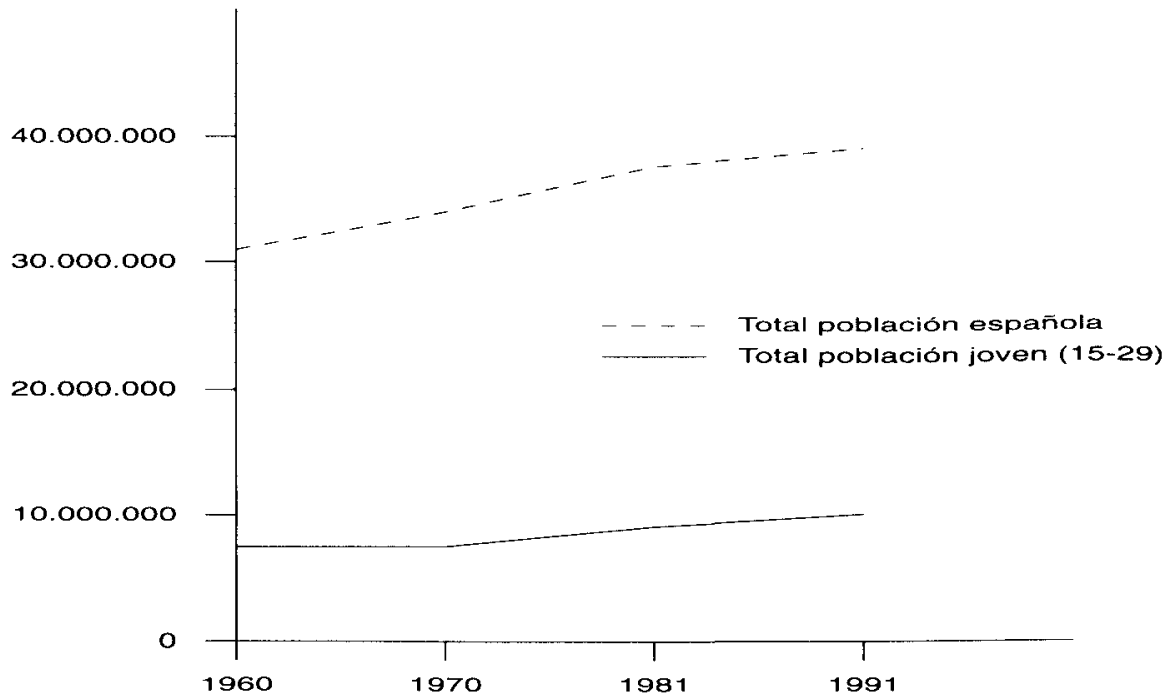

Gráfico 1. Evolución de la población total y la población joven en España, 1960-1991. 
nació entre 1941 y 1955, años de alta natalidad en todo el país, incluyendo el baby boom de la postguerra, pero que, dadas las características socioeconómicas y sanitarias de esta época de la historia de España, fueron tiempos de alta mortalidad infantil. Por el contrario, los jóvenes de 1991, nacidos entre 1961 y 1976, años en los que a pesar de que las tasas de natalidad se iban reduciendo, las mejoras socioeconómicas, y con ellas las sanitarias, posibilitaron la reducción de las tasas de mortalidad infantil, y con ello, el aumento de los que han podido llegar a las edades de juventud.

Esta evolución nos muestra que, si bien el principal factor demográfico a considerar en el volumen de jóvenes de una población es la natalidad, la incidencia de la mortalidad infantil tiene también una importancia fundamental que ha de considerase conjuntamente con aquélla.

Descendiendo ahora en la escala de estudio se observa que no todas las Comunidades Autónomas tienen proporciones similares de jóvenes. Galicia, Asturias, Cantabria, Castilla-León, La Rioja, Extremadura y Aragón tienen valores inferiores a la media nacional. Semejantes a esa cifra la tienen las de Navarra, Cataluña, Castilla-La Mancha, Valencia y Baleares. Sin embargo, hay otras: País Vasco, Madrid, Andalucía, Murcia y Canarias que superan ese valor.

En el mapa de la figura $n^{\circ} 1$ se han representado las proporciones de jóvenes alcanzadas en las distintas Comunidades Autónomas, según la escala comprendida entre el valor máximo de Canarias $(28,67)$ y el mínimo de Asturias $(22,10 \%)$.

Esta distribución espacial, en la que se pueden observar unas claras diferencias entre Norte y Sur así como entre interior y litoral, hace pensar en causas derivadas de la propia estructura de la población, que llevan consigo un determinado comportamiento de la misma. Es decir, en aquellas Comunidades Autónomas en las que los grupos de población en edades de procrear sean numerosos, la tasa de natalidad será mayor - aunque sea baja como tendencia general - que en aquellas otras en las que los citados grupos tengan un volumen escaso. La proporción de jóvenes será también mayor en aquellas regiones en las que la tasa de mortalidad general sea más alta, debido a que la proporción de adultos y viejos será menor, por lo que el porcentaje que representen los jóvenes, dentro de la misma, será mayor. También la tasa de mortalidad infantil de los distintos ámbitos espaciales es un factor de indudable importancia, pero, en este caso, ya no será una consecuencia de la estructura de la población, sino del nivel de desarrollo socioeconómico y cultural de cada comunidad, que se reflejará en los adelantos y mejoras de las costumbres sanitarias. 


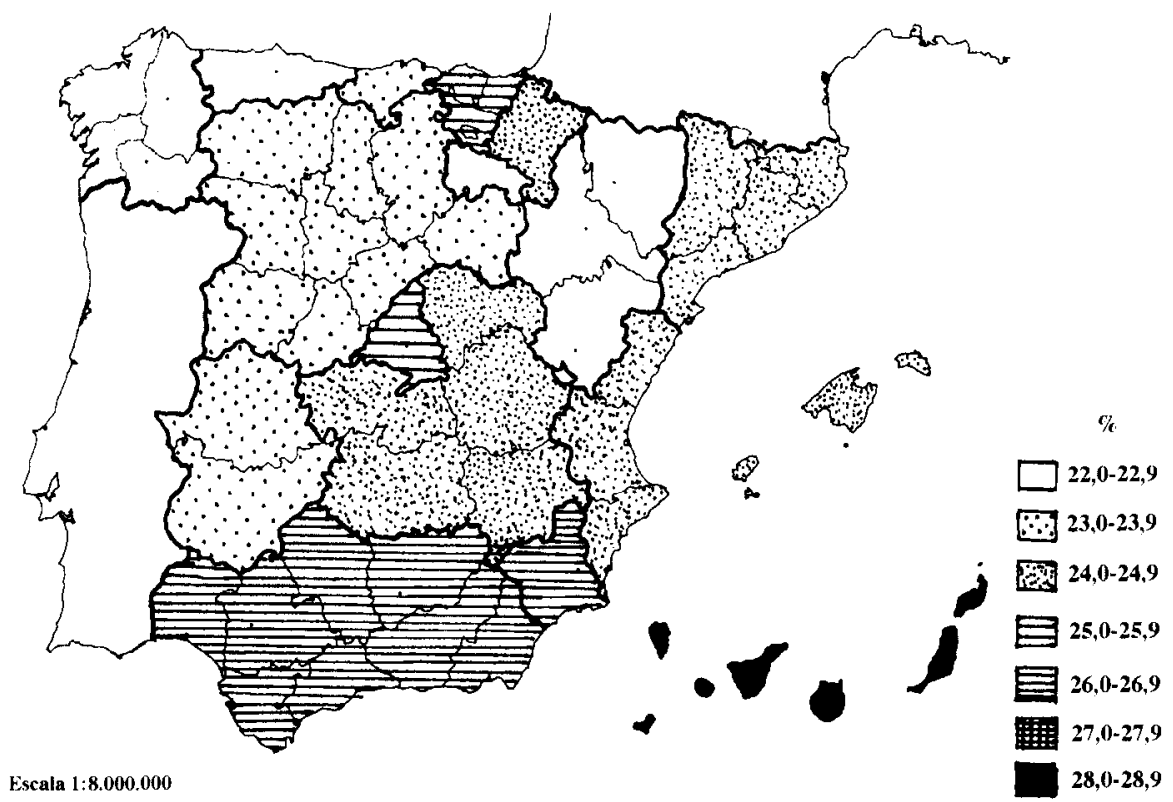

Fig. 1. Porcentaje de población joven (15-29 años) sobre el total de la población por Comunidades Autónomas.

Fuente: Juventud en cifras, 1992.

D. General de la Juventud.

En otro nivel de análisis, los movimientos migratorios entre Comunidades Autónomas tienen una clara influencia, tanto en la estructura de la población como en el porcentaje de jóvenes que tenga cada una de ellas. Es una ley generalmente admitida que, en todo el mundo, los jóvenes son los que más emigran, o dicho de otro modo, del total de emigrantes mundiales la mayoría son jóvenes. Por eso las regiones que tengan saldos migratorios positivos verán aumentar su proporción de personas comprendidas entre los 15 y los 29 años; por el contrario, aquéllas que lo tengan negativo verán disminuir dicho porcentaje, y, como consecuencia de ello, su tasa de natalidad decrecerá y aumentará la de mortalidad, al quedar la población más envejecida.

Por último, son razones de tipo económico las que subyacen bajo los factores que acabamos de analizar. Las regiones con mayor nivel de renta serán las que atraigan a esos emigrantes procedentes de las menos favorecidas, y las de menor desarrollo económico serán las que verán salir de su región a esa población joven. 
Sin embargo, la tendencia general mundial es que las áreas más desarrolladas son las que tienen menor tasa de natalidad $y$, en un estadio evolucionado de la transición demográfica, son las que ven aumentar un poco la tasa de mortalidad, a causa del envejecimiento de su población. Esta espiral de causas y consecuencias concatenadas resultan un tanto elementales si se analizan por separado, por lo que hay que pensar en su íntima relación si se quiere entender tanto la actual estructura de la población de las diferentes Comunidades Autónomas como la proporción de jóvenes que encontramos en cada una de ellas.

Veamos ahora, de forma somera, cuál es la influencia de cada uno de los factores que acabamos de señalar en las diferentes proporciones de población juvenil y en las distintas regiones españolas actuales.

Las Comunidades Autónomas con mayor proporción de jóvenes, en 1991, eran, por este orden, Canarias, Andalucía y Murcia, regiones en las que todas las circunstancias demográficas pueden unirse para explicar esos elevados porcentajes de jóvenes. En efecto, son las que tienen mayor tasa de natalidad de entre todas las regiones españolas, en 1965. Tienen baja tasa de mortalidad infantil, y aunque la tasa de mortalidad general, en ese mismo año, era de las más bajas, se debía a una estructura de población todavía muy joven (Del CAmpo, S., y NAvarro López, M. 1987). La emigración, sin embargo, es, en el caso de Murcia y Andalucía, un factor negativo, puesto que era todavía muy numerosa en aquellos momentos (Anuario Estadístico de 1982). En el caso canario, el saldo migratorio era positivo, lo que viene a sumarse a los aspectos netamente demográficos; además, a ello se une su carácter insular y la lejanía de la península, que puede dificultar la emigración de esos jóvenes. Autonomías como las de Madrid, Cataluña o País Vasco, con alta natalidad, baja mortalidad infantil y baja mortalidad general, tienen en el factor inmigración la causa más destacada, que se explica por ser las tres de mayor renta per cápita en esos años (Banco de Bilbao).

En el caso contrario se hallan las Comunidades Autónomas que tienen menor proporción de jóvenes. En ese mismo año y de mayor a menor porcentaje, estaban las de: Aragón, Galicia, La Rioja y Asturias. También en este caso, pero con carácter negativo, los mismos factores demográficos y migratorios van a influir en la explicación de esos porcentajes. Son las cuatro regiones con menor tasa de natalidad en 1965, y aunque el orden difiere, pues a Galicia con una tasa del $17,42 \%$, le sigue Aragón con un $17,59 \%$, en tercer lugar se encuentra la región asturiana con un $17,73 \%, y$, por último, La Rioja presenta una tasa del $18,06 \%$, las diferencias son mínimas. Sus tasas de mortalidad infantil eran relativamente elevadas, ocu- 
pando Aragón, el noveno lugar entre todas las regiones españolas, Asturias el décimo, y el decimotercero y decimoquinto eran los de Galicia y La Rioja respectivamente; ello viene a sumarse al efecto de la baja natalidad. Las tasas de mortalidad general eran también relativamente elevadas, a excepción de la asturiana que era de las más bajas de España. Se observa de nuevo la gran influencia de la estructura por edad de estas regiones, bastante envejecidas, que lleva consigo un comportamiento natural de la población como el que acabamos de señalar (DEL CAMPO, S., Y NAVARRO LóPEZ, M., 1987). La emigración supone en todas ellas un factor que debe también sumarse, ya que todas son focos de emigración (Anuario Estadístico de 1982). La explicación, sin lugar a dudas, se debe al bajo lugar que ocupan en la renta per cápita estas regiones españolas, que a excepción de la de La Rioja, podían considerarse bajas (Banco de Bilbao).

Después de este planteamiento general, que consideramos básico en la explicación de la distribución espacial de la proporción de jóvenes entre las distintas regiones españolas, habría que profundizar más aún, en su estudio, para saber en qué medida influye cada una, propósito que dejamos para el próximo número de la revista. En él se estudiará, ya no sólo a escala de las Comunidades Autónomas, sino de las provincias, la influencia de la estructura por edades de la población, de las tasas de natalidad, mortalidad infantil y general, movimientos migratorios, nivel de renta per cápita, asi como de otros indicadores económicos que se puedan incluir para su explicación. 


\section{BIBLIOGRAFÍA}

BANCO DE BILBAO (1984): «Renta nacional de España 1981 y su distribución provincial». Zamudio, Vizcaya, $272 \mathrm{pp}$.

Campo Urbano, S. del, y Navarro Lopez, M. (1987): «Nuevo Análisis de la Población Española». Ariel Sociología. Barcelona. 267 pp.

I.N.E.: "Censos de la Población de España».

García Barbancho, A. (1982): "Población, empleo y paro». Pirámide. Madrid. $158 \mathrm{pp}$.

Navarro López, M., y Mateo Rivas, Ma J. (1993): “Informe Juventud en España". Ministerio de Asuntos Sociales. Instituto de la Juventud. Madrid. $275 \mathrm{pp}$.

Navarro López, M., y Mateo Rivas, Ma J. (1993): "Juventud en Cifras 1992". Ministerio de Asuntos Sociales. Instituto de la Juventud. Madrid. $122 \mathrm{pp}$. 